\title{
Fuzzy-controlled Power-aware Routing Protocol (FPRP) for Mobile Ad Hoc Networks
}

\author{
Anuradha Banerjee \\ Dept. of Computer Applications \\ Kalyani Govt. Engg. College \\ West Bengal, India
}

\author{
Dr. Paramartha Dutta \\ Dept. of Computer and System Sciences \\ Visva-Bharati University, Santiniketan \\ West Bengal, India
}

\begin{abstract}
In multi-hop wireless ad-hoc networks, designing energy-efficient routing protocols is critical since nodes are power-constrained. However, it is also an inherently hard problem due to two important factors: First, the nodes may be mobile, demanding the energy-efficient routing protocol to be fully distributed and adaptive to the current states of nodes; second, the wireless links may be uni-directional due to asymmetric power configurations of adjacent nodes. In this paper, I propose a fuzzy-controlled poweraware routing protocol (FPRP) that dynamically makes local routing decisions so that a near-optimal power-efficient end-toend route is formed for forwarding data packets. The protocol is fully distributed such that only location information of neighboring nodes are exploited in each routing node. Simulation results firmly establish the effectiveness of the protocol.
\end{abstract}

\section{General Terms}

Ad hoc networks, routing protocol.

\section{Keywords}

Ad Hoc Network, fuzzy controller, power-awareness, lifetime status, router.

\section{INTRODUCTION}

A mobile ad hoc network is a collection of mobile nodes that communicate with each other without the aid of an established infrastructure or centralized administration. Its applications range from civilian use to emergency rescue sites and battlefields. The communication in ad hoc networks is either single hop or multihop. If a node $n_{j}$ resides within the radio-range or transmission range of another node $n_{i}$, then it can directly receive messages from $n_{i}$ and the communication will be termed as single hop. Otherwise, a chain of intermediate nodes need to establish a multi-hop bridge between the source and destination nodes. Those intermediate nodes are often termed as routers [1-6].

Several power aware routing protocols have been proposed for ad hoc networks. In reference [2], maximum residual packet capacity (MRPC) routing is proposed. Given the battery power level at all nodes, the MRPC algorithm [2] selects the path that maximizes the total number of packets that may be ideally transmitted. Such a path should exist for a long time; otherwise it is most likely not to transmit more packets. Hence, MRPC could be put under the category of stability based routing. Let the battery power of a node $n_{i}$ at a certain time instant be $B_{i}$. Let $E_{i, j}$ be the transmission energy required by node $n_{i}$ to transmit a packet over link $\left(n_{i}, n_{j}\right)$ to node $n_{j}$. Let $r$ be a route between the source $n_{s}$ and destination $n_{d}$ and it includes the link $\left(n_{i}, n_{j}\right)$. Assuming all other flows sharing the path $r$ do not transmit any further traffic, the maximum number of packets node $\mathrm{n}_{\mathrm{i}}$ can forward over the link $\left(n_{i}, n_{j}\right)$ to node $n_{j}$ is defined as the node-link metric $C_{i, j}=B_{i} / E_{i, j}$. The maximum lifetime of the route $r$ is determined by the weakest intermediate node i.e. the one with the least $C_{i, j}$ value. Life $_{r}=$ $\operatorname{Min}\left\{C_{i, j}\right\}$ where the link $\left(n_{i}, n_{j}\right) \in$ r. If $Q$ is the set of all available $\mathrm{n}_{\mathrm{s}}-\mathrm{n}_{\mathrm{d}}$ routes, the MRPC protocol selects the route $\mathrm{k} \in \mathrm{Q}$ such that Life $_{\mathrm{k}}=\operatorname{Max}\left\{\right.$ Life $\left._{\mathrm{r}} \mid \mathrm{r} \in \mathrm{Q}\right\}$. the protocol minimum battery cost routing (MBCR) [3] aims for a route with the maximum remaining battery capacity. Let the battery power of a node $n_{i}$ at a certain time instant be $\mathrm{B}_{\mathrm{i}}$. The battery cost function $\mathrm{f}(\mathrm{i})$ at that node is given by, $f(i)=1 / B_{i}$. The battery cost $C_{r}$ of a route $r$ between the source $n_{s}$ and destination $n_{d}$ consisting of 1 nodes is given by,

$1-1$

$\mathrm{C}_{\mathrm{r}}=\sum \mathrm{f}(\mathrm{i})$

$\mathrm{i}=0$

If $\mathrm{Q}$ is the set of all available $\mathrm{n}_{\mathrm{s}}-\mathrm{n}_{\mathrm{d}}$ routes, the MBCR protocol selects the route $k \in Q$ such that $C_{k}=\operatorname{Min}\left\{C_{r} \mid r \in Q\right\}$.

The min-max battery cost routing (MMBCR) [4] assigns the battery power of a route to the minimum residual battery power of a node (bottleneck node) along the route. The desired route is then the route with the maximum battery power. If there is a tie, MMBCR chooses the route with the shortest hop count. When all nodes in the network have almost identical residual battery power, MMBCR would result in frequent route changes. This is because the algorithm is sensitive to even slight changes in the residual battery power of the nodes and path selection often has to be done using the secondary criteria of hop count. When nodes have fairly different residual battery power, MMBCR would result in less frequent route changes. This is because MMBCR chooses nodes that have a larger residual battery power and these nodes are more likely to survive for a long time in comparison to nodes that have a lesser residual battery power.

The minimum transmission power routing MTPR [5] considers the energy consumed per hop $\left(n_{i}, n_{j}\right)$ from node $n_{i}$ to node $n_{j}$ as the link metric. The total transmission power $P_{r}$ of a route $r$ between the source $n_{s}$ and destination $n_{d}$ consisting of 1 nodes is given by,

$1-1$

$\mathrm{P}_{\mathrm{r}}=\sum \mathrm{P}\left(\mathrm{n}_{\mathrm{i}}, \mathrm{n}_{\mathrm{i}+1}\right)$

$\mathrm{i}=0$

where $P\left(n_{i}, n_{i+1}\right)$ is the power that $n_{i}$ spends in transmitting a packet to $n_{i+1}$ in route $r ; n_{0}=n_{s}, n_{l}=n_{d}$ and for all $i, n_{i} \in r$. If $Q$ is 
the set of all available $n_{s}-n_{d}$ routes, the MBCR protocol selects the route $\mathrm{k} \in \mathrm{Q}$ such that $\mathrm{P}_{\mathrm{k}}=\operatorname{Min}\left\{\mathrm{P}_{\mathrm{r}} \mid \mathrm{r} \in \mathrm{Q}\right\}$

Yuan Xue et. Al. proposed a location aided power-aware routing (to be referred to as LPR in this article subsequently) protocol in ad hoc networks in [6]. It instructs each node to adjust its transmission power so that the power is just sufficient to reach the desired downlink neighbor. It adopts a greedy algorithm to determine the relay region of neighbors. Particularly, if $n_{s}$ is the sender and $n_{d}$ is the destination, $n_{s}$ first computes and merges the relay regions of its neighbors. The relay regions of $n_{s}$ divide the entire two dimensional region into multiple sections. The routing decision is made depending on which section covers the location of $n_{d}$.

In the present article, I propose a fuzzy controlled power aware routing protocol (FPRP) that considers various parameters of lifetime of a node and combines them according to a fuzzy controller named route decider (RD). This controller is embedded in every node which incorporates intelligence. Among the various paths through which route-requests arrive at the destination, the best path is selected depending upon the lifetime status of their routers. The parameters of lifetime status of a node are its remaining energy, rate of energy depletion, the number of communication routes already established through it and distance between consecutive routers. Below mentioned practical observations in respect of ad hoc networks, stimulate the design of RD.

i) Higher the residual battery power of a node, higher is its remaining lifetime.

ii) Lesser the rate of energy depletion of a node, higher is its remaining lifetime.

iii) Lesser the distance between consecutive routers, higher is the lifetime of the route.

iv) Lesser the number of established communication routes passing through a node, lesser is the chance of its energy depletion and as a result, its lifetime increases.

Reasons for choosing fuzzy logic are that fuzzy logic is flexible, easy to understand and tolerant of imprecise data. Moreover, it is based on natural language, can be blended with conventional control techniques and can efficiently model non-linear functions of arbitrary complexity.

Simulation results presented in section IV firmly establish the effectiveness of FPRP compared to other state-of-the-art power aware routing protocols in ad hoc networks.

\section{INPUT PARAMETERS OF FUZZY CONTROLLER RD}

The input parameters of RD are residual energy index, energy depletion index, communication load and proximity index. Below they are described in detail.

\section{Residual Energy Index}

The Residual energy index of a node $n_{i}$ at time $t$ is denoted as $\alpha_{i}(t)$ and defined as,

$$
\alpha_{\mathrm{i}}(\mathrm{t})=1-\mathrm{e}_{\mathrm{i}}(\mathrm{t}) / \mathrm{E}_{\mathrm{i}}
$$

where $e_{i}(t)$ and $E_{i}$ indicate the consumed battery power at time $t$ and maximum battery capacity of $n_{i}$, respectively. It may be noted from the formulation in (1) that $0 \leq \alpha_{\mathrm{i}}(\mathrm{t}) \leq 1$. Values close to 1 enhance capability of $\mathrm{n}_{\mathrm{i}}$ as a router.

\section{Energy Depletion Index}

The energy depletion index of a node $n_{i}$ at time $t$ is denoted as $\beta_{\mathrm{i}}(\mathrm{t})$ and defined as,

$\beta_{i}(t)= \begin{cases}\left(\mathrm{t} \mathrm{e}_{\mathrm{i}}(\mathrm{t})\right) /\left(\left(\mathrm{t}-\mathrm{t}_{\mathrm{i}}\right) \mathrm{E}_{\mathrm{i}}\right) & \text { if }\left(\mathrm{t} \mathrm{e}_{\mathrm{i}}(\mathrm{t})\right) /\left(\left(\mathrm{t}-\mathrm{t}_{\mathrm{i}}\right) \mathrm{E}_{\mathrm{i}}\right)<1 \\ & \text { otherwise }\end{cases}$

Significance of $e_{i}(t)$ and $E_{i}$ has been mentioned earlier. $t$ is the current time and $t_{i}$ is the time when $n_{i}$ started its operation in the network with full battery capacity. It may be noted from the formulation in (2) that $0 \leq \beta_{i}(t) \leq 1$. Values close to 0 enhance capability of $n_{i}$ as a router.

\section{$\underline{\text { Communication Load }}$}

The communication load of a node $\mathrm{n}_{\mathrm{i}}$ at time $\mathrm{t}$ is denoted as $\gamma_{\mathrm{i}}(\mathrm{t})$ and defined as,

$\gamma_{\mathrm{i}}(\mathrm{t})=1-1 / \mathrm{c}_{\mathrm{i}}(\mathrm{t})$

where $c_{i}(t)$ is the number of communication paths passing through $\mathrm{n}_{\mathrm{i}}$ at time $\mathrm{t}$. It may be noted from the formulation in (3) that $0 \leq$ $\gamma_{i}(t) \leq 1$. Values close to 0 enhance capability of $n_{i}$ as a router.

\section{Proximity index}

Assuming $n_{j}$ to be a downlink neighbor of node $n_{i}$ at time $t$, proximity index $\phi_{\mathrm{ij}}(\mathrm{t})$ between those two nodes at time $\mathrm{t}$ is given by,

$\phi_{\mathrm{ij}}(\mathrm{t})=1-\mathrm{D}_{\mathrm{ij}}(\mathrm{t}) / \mathrm{R}_{\mathrm{i}}$

$D_{i j}(t)$ is the distance between $n_{i}$ and $n_{j}$ at time $t$ and $R_{i}$ is the radiorange of $n_{i}$. FPRP assumes that each transmitter node can adjust it's transmit power based on its distance from the desired downlink neighbor. The lesser the distance between $n_{i}$ and $n_{j}$ (upper limit of the distance is $\mathrm{R}_{\mathrm{i}}$ ), lesser will be the power required by $n_{i}$ to send a message to $n_{j}$ if the link between these two nodes really take part in the selected communication path between the associated pair of source and destination nodes.

\section{DESIGN OF RULE BASES OF RD}

TABLE I

RANGE DIVISION OF PARAMETERS OF RD

\begin{tabular}{|l|l|l|}
\hline Range of $\alpha$ & Range of $\beta, \gamma, \phi$ and $\psi$ & Fuzzy variable \\
\hline $0-0.40$ & $0-0.25$ & a1 \\
\hline $0.40-0.60$ & $0.25-0.50$ & a2 \\
\hline $0.60-0.80$ & $0.50-0.75$ & a3 \\
\hline $0.80-1.00$ & $0.75-1.00$ & a4 \\
\hline
\end{tabular}

According to the study of discharge curve of batteries heavily 
used in ad hoc networks [1], at least $40 \%$ of total charge is required to remain in operable condition (denoted as a1), $40 \%$ to $60 \%$ is just satisfactory (a2), $60 \%$ to $80 \%$ (a3) is good while the next higher range i.e. $80 \%$ to $100 \%$ (a4) is more than sufficient. Ranges of the remaining input parameters of $\mathrm{RD}$ are divided uniformly. Also all the outputs of RD (including its temporary outputs and the final output) follow uniform range distribution between 0 and 1 .

TABLE II

FUZZY COMBINATION OF $\alpha$ AND $\beta$ PRODUCING t1

\begin{tabular}{|l|l|l|l|l|}
\hline $\begin{array}{l}\alpha \rightarrow \\
\beta \downarrow\end{array}$ & a1 & a2 & a3 & a4 \\
\hline a1 & a1 & a2 & a3 & a4 \\
\hline a2 & a1 & a2 & a3 & a4 \\
\hline a3 & a1 & a1 & a2 & a3 \\
\hline a4 & a1 & a1 & a2 & a2 \\
\hline
\end{tabular}

TABLE III

FUZZY COMBINATION OF t1 AND $\gamma$ PRODUCING t2

\begin{tabular}{|l|l|l|l|l|}
\hline $\begin{array}{l}\mathrm{t} 1 \rightarrow \\
\gamma \downarrow\end{array}$ & a1 & a2 & a3 & a4 \\
\hline a1 & a1 & a2 & a3 & a4 \\
\hline a2 & a1 & a2 & a3 & a4 \\
\hline a3 & a1 & a2 & a3 & a4 \\
\hline a4 & a1 & a1 & a2 & a3 \\
\hline
\end{tabular}

TABLE IV

FUZZY COMBINATION OF t2 AND $\phi$ PRODUCING $\psi$

\begin{tabular}{|l|l|l|l|l|}
\hline $\begin{array}{l}\mathrm{t} 2 \rightarrow \\
\gamma \downarrow\end{array}$ & a1 & a2 & a3 & a4 \\
\hline a1 & a1 & a2 & a3 & a3 \\
\hline a2 & a1 & a2 & a3 & a4 \\
\hline a3 & a1 & a2 & a3 & a4 \\
\hline a4 & a1 & a3 & a4 & a4 \\
\hline
\end{tabular}

Table II produces the fuzzy combination of $\alpha$ and $\beta . \alpha$ is given more weight because a node with high residual energy may survive for some time in spite of high rate of energy depletion but a node with residual energy as low as a1 is unable to operate irrespective of its low rate of power depletion. The temporary output produced by table II is denoted as t1. Table III produces the fuzzy combination of $\mathrm{t} 1$ and $\gamma$ generating another temporary output $\mathrm{t} 2$. The chemistry of $\mathrm{t} 2$ and $\phi$ appears in table IV producing $\psi$. In table III, $\mathrm{t} 1$ is assigned more weight than $\gamma$ and in table IV, $\mathrm{t} 2$ is assigned more importance than $\phi$. The reason is that $\mathrm{t} 1$ and $\mathrm{t} 2$ are combinations of parameters whereas $\gamma$ and $\phi$ are single parameters.

The route whose minimum lifetime status of routers, is maximum among all the available routes between a specific pair of source and destination nodes, is selected as optimal. In case of availability of more than one choice, the one with minimum number of hops is selected.

\section{SIMULATION RESULTS}

Simulation of the mobile network has been carried out using ns-2 [7] simulator on $800 \mathrm{MHz}$ Pentium IV processor, $40 \mathrm{~GB}$ hard disk capacity and Red Hat Linux version 6.2 operating system with specification of parameters mentioned in table V. Supporting graphs appear in figures 1,2 and 3 showing emphatic improvements in favor of FPRP. Number of nodes has been taken as 50, 150, 300, 500, 1000 and 2000 in six different independent simulation studies. The applied confidence interval is $95 \%$. Speed of a node is chosen randomly between 0 to $45 \mathrm{~m} / \mathrm{sec}$. Performance of the FPRP method is compared with three other most happening power-aware routing protocols, MTPR [5], MMBCR [4] and LPR [6]. In order to maintain uniformity of the implementation platform, we have used ns- 2 simulator for all the above-mentioned communication protocols and FPRP. Total number of message packets required to establish the connection between a specific pair of source and destination nodes or message overhead, packet delivery ratio and transmission delay are used as performance metrics. Their definitions are presented below.

\section{Definition 1: Mean \% of packet delivery ratio (MPR)}

Let, $\mathrm{F}^{\sim}{ }_{\mathrm{ij}}$ denotes the number of packets successfully delivered during communication sessions from $n_{i}$ to $n_{j} ; M_{i j}^{\sim}$ indicates the number of packets generated by $n_{i}$ for $n_{j}\left(n_{i}\right.$ and $n_{j}$ are any two arbitrary nodes in the network; $\mathrm{Z}$ indicates the set of all nodes in the network. Percentage of packet delivery ratio of the session $\mathrm{MPR}_{\mathrm{ij}}$ is expressed as (5).

$$
\begin{gathered}
\operatorname{MPR}_{\mathrm{ij}}=(1 /|\mathrm{Z}|) \sum\left(\mathrm{F}_{\mathrm{ij}}^{\sim} / \mathrm{M}_{\mathrm{ij}}^{\sim}\right) \times 100 \\
\mathrm{n}_{\mathrm{i}}, \mathrm{n}_{\mathrm{j}} \in \mathrm{Z}
\end{gathered}
$$

\section{Definition 2: Per Node Message Overhead (PNMO)}

Let, $\mathrm{S}_{\mathrm{ij}}$ denotes the total number of messages (including route discovery packets) transmitted during communication sessions from $n_{i}$ to $n_{j} ; Z$ indicates the set of all nodes in the network. Then, per node message overhead of the network can be expressed as (6).

$$
\begin{gathered}
\mathrm{PNMO}_{\mathrm{ij}}=(1 /|\mathrm{Z}|) \sum \mathrm{S}_{\mathrm{ij}}^{\sim} \\
\mathrm{n}_{\mathrm{i}}, \mathrm{n}_{\mathrm{j}} \in \mathrm{Z}
\end{gathered}
$$

\section{Definition 3: Per Node Delay in Seconds (PNDS)}

Let, $\mathrm{t}_{\mathrm{ij}}^{\mathrm{s}}$ and $\mathrm{t}_{\mathrm{ij}}^{\mathrm{f}}$ denote the timestamps (in seconds) of initiation and completion, respectively, of a communication session from $n_{i}$ to $\mathrm{n}_{\mathrm{j}}$. Per node transmission delay (in seconds) of the network can be expressed as (7).

$$
\begin{gathered}
\operatorname{PNDS}_{\mathrm{ij}}=(1 /|\mathrm{Z}|) \sum\left(\mathrm{t}_{\mathrm{ij}}^{\mathrm{f}}-\mathrm{t}_{\mathrm{ij}}^{\mathrm{s}}\right) \\
\mathrm{n}_{\mathrm{i}}, \mathrm{n}_{\mathrm{j}} \in \mathrm{Z}
\end{gathered}
$$


TABLE V

SIMULATION PARAMETERS

\begin{tabular}{|l|l|}
\hline Parameter & Value \\
\hline Network Area & $\begin{array}{l}900 \times 900 \mathrm{~m}^{2} \text { in first ten runs, 2000 } \times 1000 \mathrm{~m}^{2} \text { in } \\
\text { nest ten runs, } 900 \times 3000 \mathrm{~m}^{2} \text { in last ten runs }\end{array}$ \\
\hline $\begin{array}{l}\text { Transmission } \\
\text { Range }\end{array}$ & $\begin{array}{l}10-50 \mathrm{~m} \text { in first ten runs, } 30-100 \mathrm{~m} \text { in next ten } \\
\text { runs, } 10-100 \mathrm{~m} \text { in last ten runs }\end{array}$ \\
\hline $\begin{array}{l}\text { Interval between } \\
\text { consecutive } \\
\text { HELLO messages }\end{array}$ & $\begin{array}{l}20 \text { seconds for first ten simulation runs, 30 seconds } \\
\text { for next ten and } 45 \text { seconds for last ten simulation } \\
\text { runs }\end{array}$ \\
\hline Number of nodes & $50-2000$ \\
\hline MAC layer & IEEE $802.11 \mathrm{~g}$ \\
\hline PAUSE_TIME & 20 seconds \\
\hline Traffic type & Constant bit rate (128 kbps/second) \\
\hline $\begin{array}{l}\text { Maximum number } \\
\text { of retries before an } \\
\text { acknowledgement } \\
\text { is obtained }\end{array}$ & 4 \\
\hline Packet Size & $\begin{array}{l}64 \text { bytes in first ten runs, 128 bytes in next ten } \\
\text { runs, 256 bytes in last ten runs (in different } \\
\text { simulation runs) }\end{array}$ \\
\hline Bandwidth & $\begin{array}{l}1-4 \text { Mbps in first ten runs, 2 - 7 Mbps in first ten } \\
\text { runs, 1-10 Mbps in last ten runs }\end{array}$ \\
\hline Mobility model & $\begin{array}{l}\text { Random waypoint mobility model in first 10 runs, } \\
\text { Random walk mobility model in subsequent } 10 \\
\text { runs and Gaussian model in last 10 runs }\end{array}$ \\
\hline Simulation Time & $1000 \mathrm{~s}$ for each run \\
\hline
\end{tabular}

In different simulation runs, nodes move according to the "random waypoint", "random walk" and "gauss-markov" model. In random waypoint model, each node begins operation by remaining stationary for PAUSE_TIME seconds (its value is mentioned in table $\mathrm{V}$ ). It then selects a random position in the space and moves to that position at a speed distributed uniformly between 0 and maximum speed i.e. $45 \mathrm{~m} / \mathrm{s}$. When it reaches the destination, a new round of pause/ move is repeated. The random walk model was originally used to emulate the unpredictable movements of particles in physics, also referred to as Brownian motion. Random walk model is very similar to random waypoint mobility model because the node movements have strong randomness in both models. The random walk model may be thought of as a specific kind of random waypoint model with PAUSE_TIME 0 seconds. On the other hand, in gauss-markov mobility mode, the velocity of a node is assumed to be correlated

Figures 1,2 and 3 graphically illustrate the performance of FPRP compared to MTPR, MMBCR and LPR. Each plotted point is an average of 30 simulation runs. Significant enhancement of network throughput $(13.75 \%, 11.62 \%$ and 9.88\% compared to MTPR, MMBCR and LPR respectively) can be noticed in favor of our present scheme, even when number of nodes is as high as 2000. Under this circumstance, on an average, per node message overhead $(20.6 \%, 17.45$ and $12.35 \%$ compared to MTPR, MMBCR and LPR respectively) and transmission delay $(10.71 \%, 9.82$ and $7.31 \%$ compared to MTPR, MMBCR and LPR respectively) also reduce substantially. over time and modeled as a gauss-markov stochastic process.

MPR vs Number of nodes

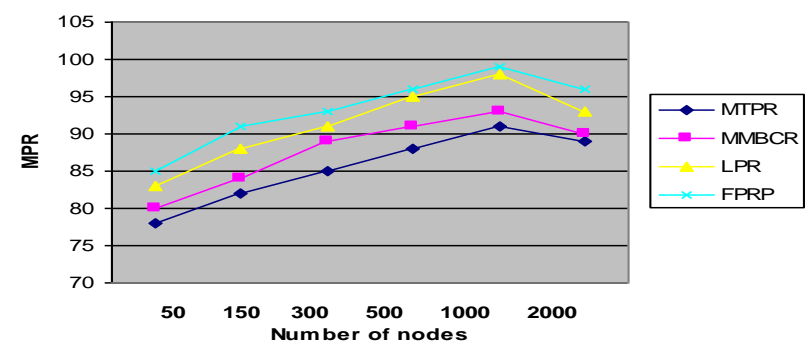

Figure 1: Graphical representation of packet delivery ratio vs number of nodes

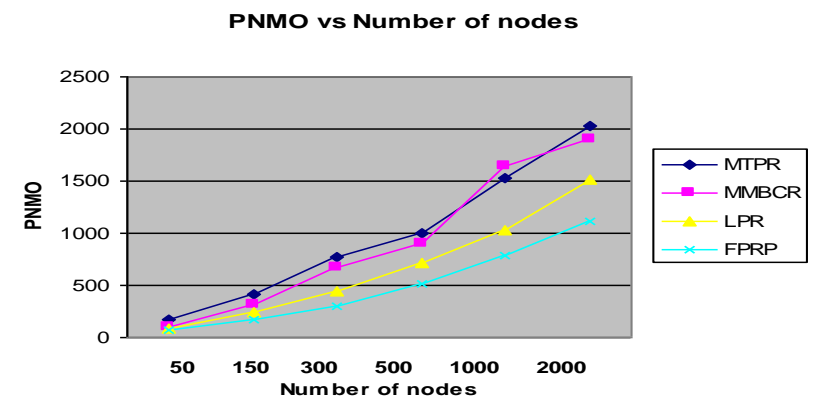

Figure 2: Graphical representation of message cost vs number of nodes

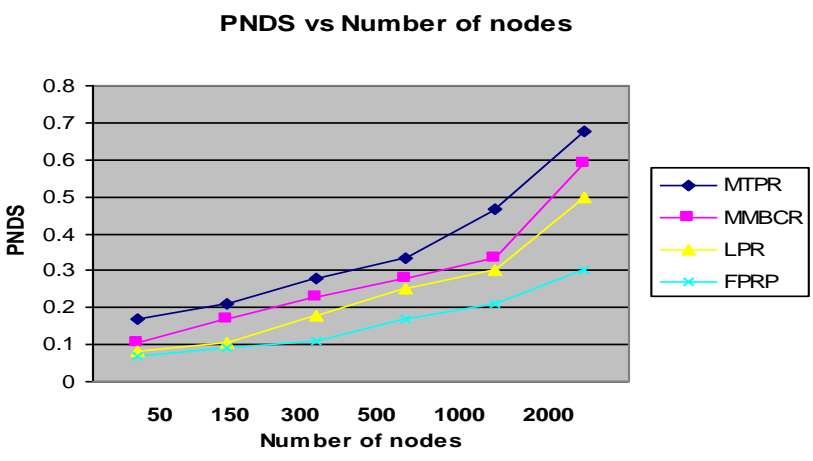

Figure 3: Graphical representation of transmission delay vs number of nodes

Reasons for such improvement in favor of FPRP are mentioned below. Since FPRP incorporates several parameters in consideration of power awareness of a route, chances of unbalanced node exhaustion and death of nodes are much lesser in FPRP than other state-of-the-art power aware routing protocols. This reduces the phenomenon of link breakage in FPRP. Link breakages inevitably give rise to flooding of route-request packets. This huge injection of route-request packets in the network again reduces battery power of nodes and increases signal collision. As a result, percentage of packets successfully delivered to respective destinations, greatly reduce. Frequent link breakage and flooding lead to wastage of time for discovering routes to destination. Therefore, transmission delay of a message from source to 
destination also increases in the above mentioned protocols (MTPR, MMBCR and LPR) compared to FPRP.

\section{CONCLUSION}

In this paper we propose a fuzzy controlled power aware routing protocol (FPRP) that is fully distributed and intelligent. It evaluates lifetime of a route based on the lifetime status of its routers. Lifetime status of routers is measured using a fuzzy controller named route decider. The fuzzy controller considers residual charge, rate of depletion, communication load and proximity of nodes. Simulation results establish that FPRP produces significant improvements than various other poweraware ad hoc network routing protocols even when the number of nodes is as high as 2000 .

\section{REFERENCES}

[1] Anuradha Banerjee, Paramartha Dutta, Fuzzycontrolled Adaptive and Intelligent Route (FAIR) Selection in Mobile Ad Hoc Networks, European Journal of Scientific Research, vol. 45, no. 3, 2010
[2] A. Misra and S. Banerjee, MRPC: Maximizing Network Lifetime for Reliable Routing in Wireless Environments, Proceedings of WCNC, 2002

[3] C.-K. Toh, "Maximum Battery Life Routing to Support Ubiquitous Mobile Computing in Wireless Ad Hoc Networks," IEEE Communications Magazine, June 2001

[4] Murthy, S. and J.J. Garcia-Luna-Aceves, An Efficient Routing Protocol for Wireless Networks, ACM Mobile Networks and App. J., Special Issue on Routing in Mobile Communication Networks, Oct. 1996, pp. 183-97

[5] Natarajan Meghanathan, Survey and taxonomy of unicast routing protocols for mobile ad hoc networks, International journal of applications of graph theory in wireless ad hoc and sensor networks, vol 1 no. 1, Dec 2009

[6] N. Meghanathan, "Energy Consumption Analysis of the Stable Path and Minimum Hop Path Routing Strategies for Mobile Ad hoc Networks," International Journal of Computer Science and Network Security, Vol. 7, No. 10, pp. 30 - 39, October 2007 